\title{
OPEN Effects of different prosthetic instrumentations on tibial bone resection in total knee arthroplasty
}

\author{
Yufeng $\mathrm{Lu}^{1}$, Xuechao Yuan ${ }^{3}$, Feng Qiao ${ }^{1}$ \& Yangquan $\mathrm{Hao}^{2 凶}$
}

Our aim was to assess the accuracy of the obtained posterior tibial slope (PTS) with a fixed angle cutting block. 247 TKAs in 213 patients were reviewed. We included 104 Legion Prosthesis, 76 U2 Knee Prosthesis, 46 NexGen LPS-Flex Prosthesis, and 21 Vanguard Knee System products. Preoperative and postoperative PTS were measured via expanded lateral tibia radiographs. For postoperative PTS, the Legion group had significantly smaller slopes than the U2 Knee group and Vanguard group. However, there was no significant difference between the Legion and NexGen groups, and no significant difference among the NexGen, U2 Knee, and Vanguard groups. Multiple linear regression showed that the different tibial lengths and preoperative PTS had statistically significant effects on postoperative PTS. However, there were weak correlations between the tibial length and PTS, and between preoperative and postoperative PTS. For TKA, although the PTS is not completely consistent with the angle of the cutting block, using conventional tibial bone resection technology with different tibial cutting instrumentations provided by various manufacturers in TKA can obtain safe PTS.

In total knee arthroplasty (TKA), the alignment of the sagittal plane of the prosthesis is as important as that of the coronal plane and axial position. Poor alignment can lead to early failure of the prosthesis ${ }^{1,2}$. The posterior tibial slope (PTS) relates to the postoperative range of motion ${ }^{3}$ and function of the extensor mechanism ${ }^{4}$. PTS also impacts tibial insert wear ${ }^{5}$ and loosening ${ }^{6}$, as well as the stability of the TKA ${ }^{7}$. Previous studies ${ }^{8-10}$ have suggested that the postoperative PTS should range from $0^{\circ}$ to $10^{\circ}$ to guarantee optimal prosthesis function. However, some authors ${ }^{11-13}$ recently recommended the reconstruction of the native PTS, depending on the intraoperative mobility and stability of the knee joint.

For TKA tibial bone resection, various manufacturers provide cutting blocks with certain PTS. In our institution, the commonly used TKA instrumentations are provided by Smith \& Nephew, United Orthopedic, Zimmer, and Biomet with angles of $3^{\circ}, 5^{\circ}, 7^{\circ}$, and $0^{\circ}$ respectively.

In the current study, we aimed to assess the PTS after different tibial cutting instrumentations were employed for TKA. We hypothesized that the PTS achieved after osteotomy with different extramedullary guidance jigs are inconsistent with the fixed angle of the cutting blocks.

\section{Patients and methods}

The protocols described herein were approved by the ethics reviewing council of Honghui Hospital, Xi'an Jiaotong University, which abides by the Declaration of Helsinki on Ethical principles for medical research involving human subjects (IRB Approval Number 202003058). Written informed consent was obtained from all participants.

This study retrospectively reviewed 320 TKAs performed by the senior surgeon (HY) using posterior-stabilizing prostheses between January 1, 2018, and December 31, 2019. The inclusion criteria were as follows: (1) preoperative diagnosis of knee osteoarthritis; and (2) true knee lateral radiographs, including at least $20 \mathrm{~cm}$ of the tibia. Exclusion criteria included (1) evidence of trauma, infection, tumor, or any congenital disorder; and (2) tibial plateau with severe bone $\operatorname{defect}(\mathrm{s})$.

Using these criteria, 247 knees of 213 patients ( 155 women and 58 men) were included. There were 122 left knees and 125 right knees. The mean patient age at the time of index operation was 62.5 years (range, 30-87 years). The mean follow-up was 15.3 months (range, 6-24 months). There were 104 Legion Prostheses

\footnotetext{
${ }^{1}$ Department of Integrated Traditional Chinese Medicine (TCM) and Western Medicine Orthopedics, Honghui Hospital, Xi'an Jiaotong University, Xi'an 710054, Shaanxi, People's Republic of China. ${ }^{2}$ Osteonecrosis and Joint Reconstruction Ward, Department of Joint Surgery, Honghui Hospital, Xi'an Jiaotong University, Xi'an 710054, Shaanxi, People's Republic of China. ${ }^{3}$ Shaanxi University of Chinese Medicine, Xianyang 712046, Shaanxi, People's Republic of China. ${ }^{\square}$ email: haoyq2008@yahoo.com
} 

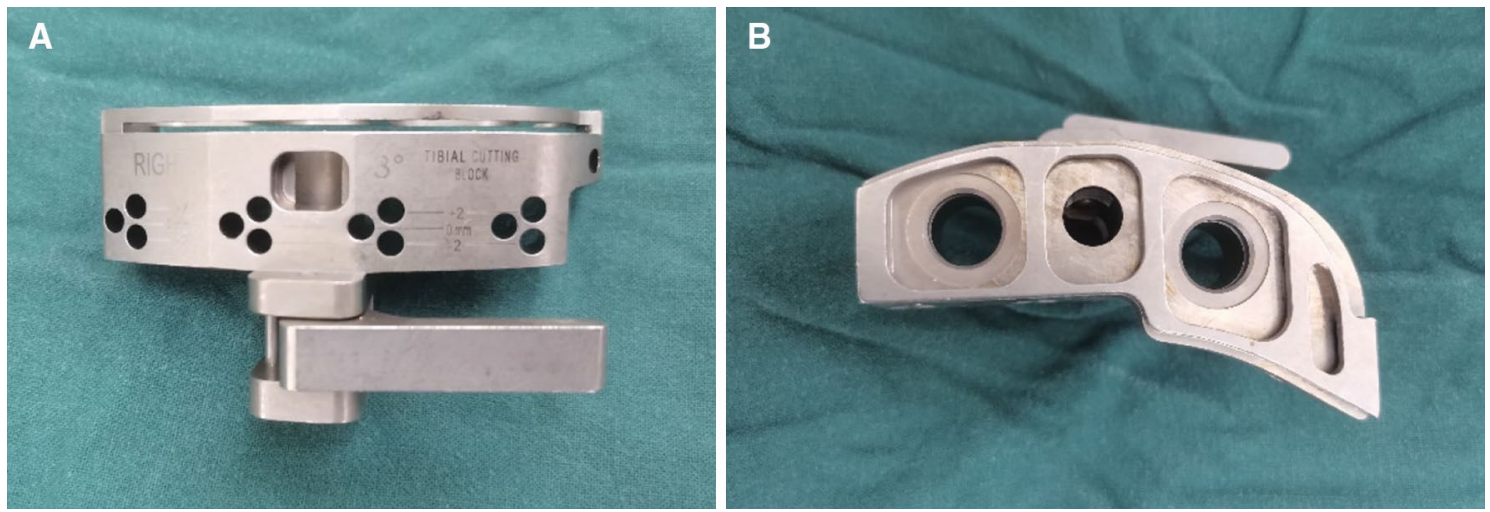

Figure 1. (A,B) Tibial cutting block provided by Smith \& Nephew has an angle of posterior inclination of $3^{\circ}$ for the Legion system.
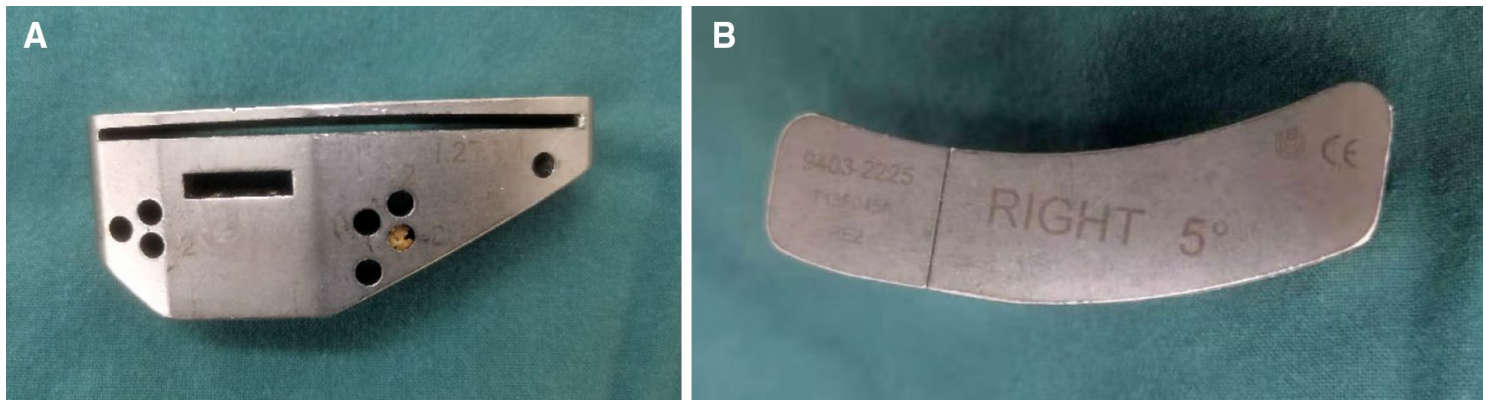

Figure 2. (A,B) Tibial cutting block provided by United Orthopedic has an angle of posterior inclination of $5^{\circ}$ for the U2 Knee system.

(Smith \& Nephew, Memphis, TN), 76 U2 Knee Prostheses (United Orthopedic, Taipei, Taiwan), 46 NexGen LPS-Flex Prostheses (Zimmer, Warsaw, IN), and 21 Vanguard Knee Systems (Biomet, Warsaw, IN) used.

Surgical technique. All TKAs were performed by the senior surgeon (HY) using a midline skin incision and medial parapatellar arthrotomy. The prosthesis was chosen by the patient according to their own situation, and none of the PTS of patients were measured before surgery. The tibia cuts were made with the use of an extramedullary guidance jig. After resection of the distal femur, the tibia was anteriorly subluxated, and the tibial alignment guide and cutting block were assembled. The guide spike was anchored to the ACL attachment of the tibia. The cutting jig was fixed to the proximal tibia by two parallel pins and one oblique pin. In the coronal plane, the alignment guide pointed to the second metatarsal, and the proximal tibia was cut perpendicular to the guide in the coronal plane. In the sagittal plane, the surgeon used his fingers to determine the PTS osteotomy. At the lower edge of the tibial tuberosity, the anterior side of the tibia was two fingers' width from the guide, and, at the upper side of the ankle joint clamp, the anterior tibial skin was three fingers' width from the guide rod. Each tibial cutting block provided by the manufacturers had an angle of posterior inclination, which was $3^{\circ}$ for the Legion system (Fig. 1A,B), $5^{\circ}$ for the U2 Knee system (Fig. $2 \mathrm{~A}, \mathrm{~B}$ ), $7^{\circ}$ for the NexGen system (Fig. $3 \mathrm{~A}, \mathrm{~B}$ ), and $0^{\circ}$ for the Vanguard system (Fig. 4,B).

All measurements were carried out with a picture archiving and communication system (PACS, Synapse, Fujifilm Inc., Tokyo, Japan). (1) PTS was measured according to Faschingbauer's method ${ }^{14}$ using the true knee lateral radiographs. The anatomic axis of the tibia was taken as the line connecting the midline of the anterior and posterior (AP) cortical edges $6 \mathrm{~cm}$ and $16 \mathrm{~cm}$ distal from the tibial plateau. The AP axis of the tibial plateau was the line connecting the AP edges of the tibial plateau. If there was an obvious osteophyte on the AP edge of the tibial plateau, the medial plateau was used as the AP axis. The preoperative PTS was $90^{\circ}$ minus the angle between the two axes (Fig. 5). The postoperative PTS was $90^{\circ}$ minus the angle between the anatomic axis of the tibia and the AP axis of the tibial component (Fig. 6). (2) The tibial component coronal alignment angle (TCCA) was defined as the angle between the mechanical axis of the tibia and transverse axis of the tibial component on postoperative standing full-length AP radiographs (Fig. 7). (3) The tibia length was defined as the distance between the midpoint of the proximal tibial articular surface and the midpoint of the distal tibial articular surface on preoperative standing full-length radiographs (Fig. 8).

The measurement data were divided into four groups according to prosthesis type: Legion group, U2 Knee group, NexGen group, and Vanguard group. All measurements were performed by two blinded observers (LY and YX) using radiographs. After 3 weeks, 20 randomly selected patients were measured again for the determination of intra-rater and inter-rater reliability. 

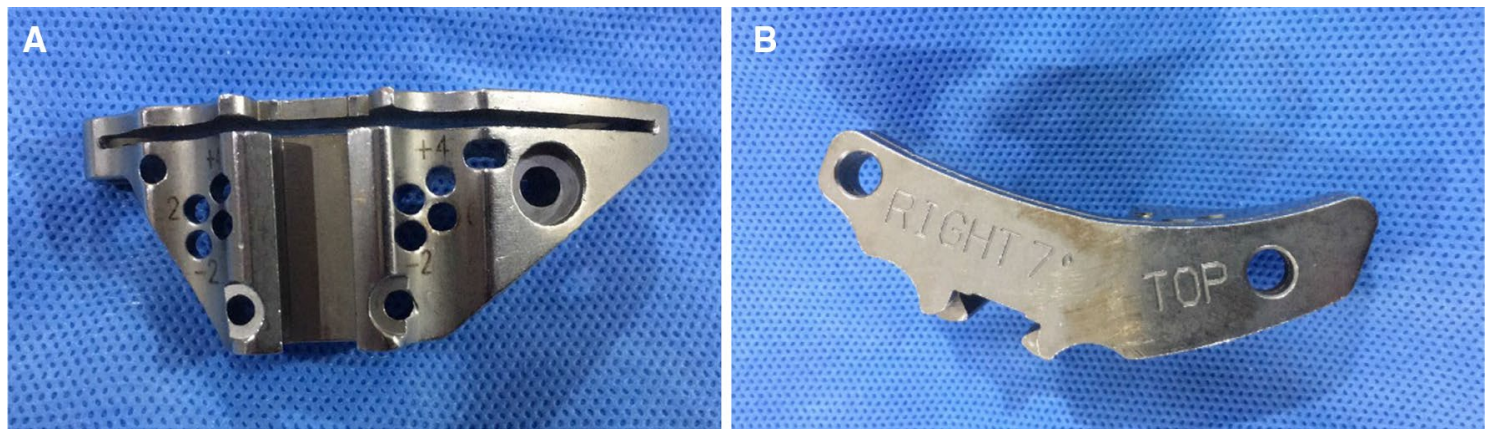

Figure 3. (A,B) Tibial cutting block provided by Zimmer has an angle of posterior inclination of $7^{\circ}$ for the NexGen system.
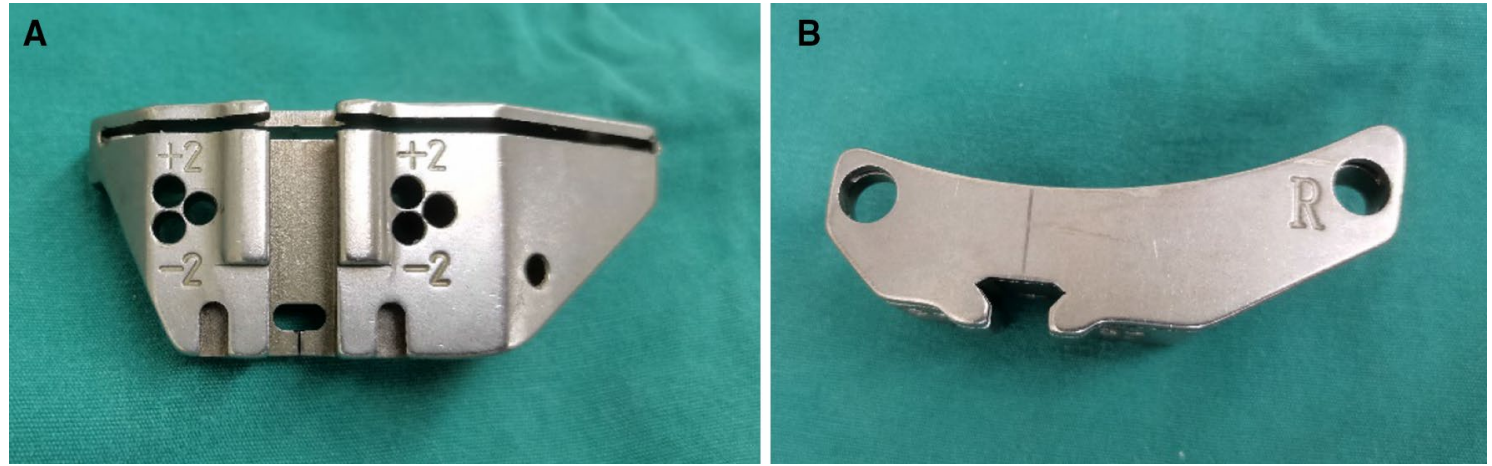

Figure 4. (A,B) Tibial cutting block provided by Biomet had an angle of posterior inclination $0^{\circ}$ for the Vanguard system.

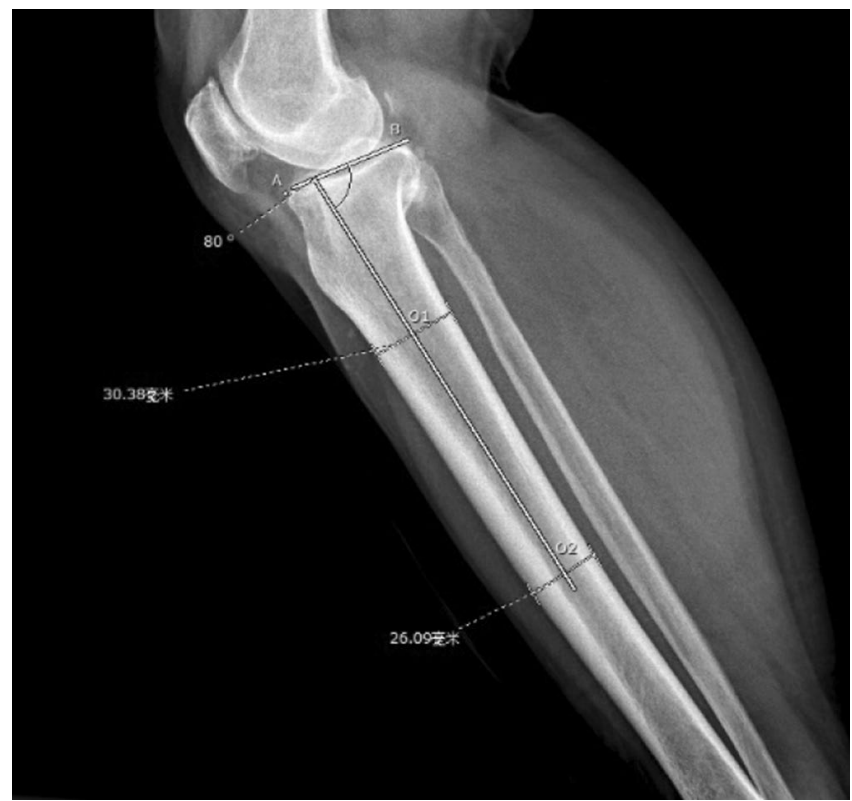

Figure 5. $\mathrm{A}$ is the anterior edge of the tibial plateau, $\mathrm{B}$ is the posterior edge of the tibial plateau, $\mathrm{O} 1$ and $\mathrm{O} 2$ are the midpoints of the anterior and posterior cortical edges $6 \mathrm{~cm}$ and $16 \mathrm{~cm}$ distal from the tibial plateau respectively. The preoperative $\mathrm{PTS}$ is $90^{\circ}$ minus the angle between the line $\mathrm{AB}$ and the line $\mathrm{O} 1 \mathrm{O} 2$. 


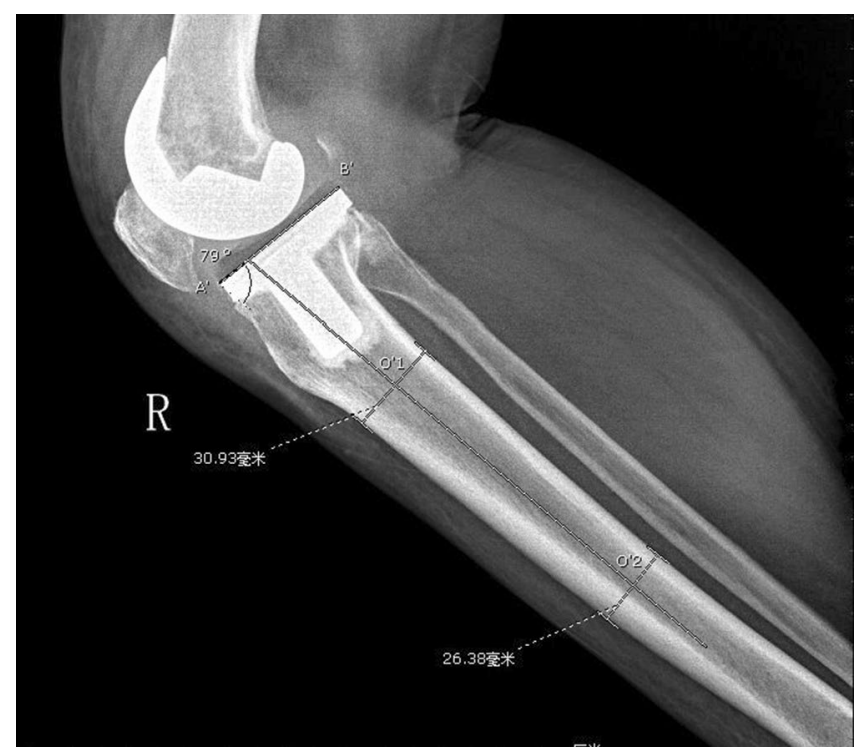

Figure 6. A' is the anterior edge of the tibial component, B' is the posterior edge of the tibial component, O'1and O'2 are the midpoints of the anterior and posterior cortical edges $6 \mathrm{~cm}$ and $16 \mathrm{~cm}$ distal from the tibial component respectively. The postoperative PTS is $90^{\circ}$ minus the angle between the line A'B' and the line O'1O'2.

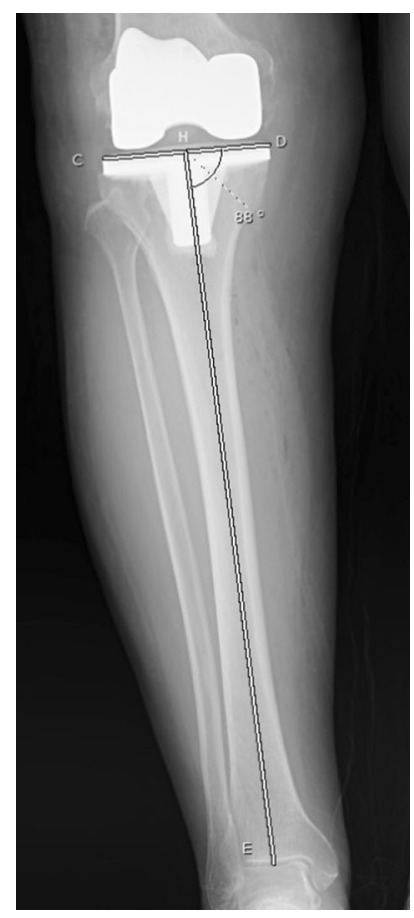

Figure 7. $\mathrm{C}$ is the lateral edge of the tibial component, $\mathrm{D}$ is the medial edge of the tibial component, $\mathrm{H}$ is the midpoint of the $\mathrm{CD}, \mathrm{E}$ is the center of the ankle joint, and the angle between $\mathrm{CD}$ and $\mathrm{HE}$ is the tibial component coronal alignment.

Clinical outcome assessment used the knee social score (KSS) pre-operatively and at final follow-up. The KSS comprises two parts: a knee score, which includes pain, stability, and range of motion (ROM) and a function score, which includes the patient's ability to walk and climb stairs, and the need for ambulatory aids.

Quantitative data were expressed as means \pm standard deviation (SD). Statistical analyses were performed using the PASW statistics 18 (SPSS Inc., Chicago, IL, USA).

The normality assumption of our data was validated by the Kolmogorov-Smirnov test. A one-way ANOVA test and Kruskal-Wallis non-parametric tests were used to compare the data for the four groups. Intra- and inter-rater reliability ${ }^{15}$ were determined using the intraclass correlation coefficient (ICC). Multiple linear regression analysis was used to investigate the possibility of association of age, sex, body side, TCCA, tibial length, 


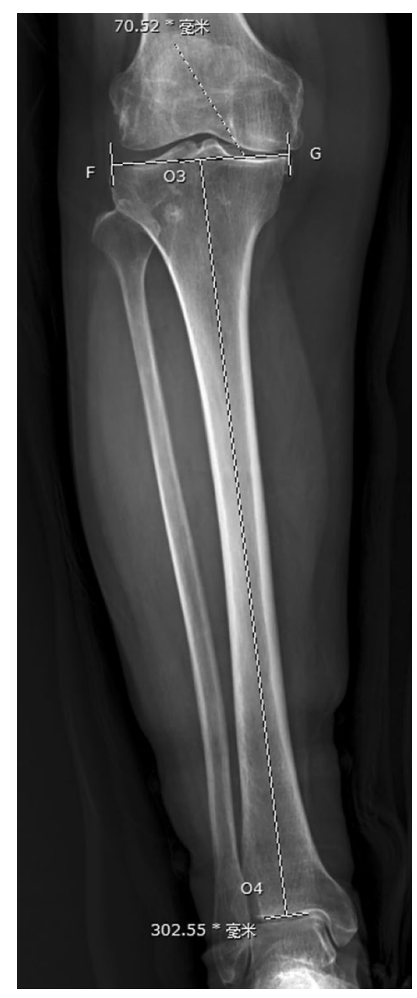

Figure 8. $\mathrm{F}$ is the lateral edge of the tibial plateau, $\mathrm{G}$ is the medial edge of the tibial plateau, $\mathrm{O} 3$ is the midpoint of FG, $\mathrm{O} 4$ is the midpoint of the distal tibial articular surface, and the length of $\mathrm{O} 3 \mathrm{O} 4$ is the tibia length.

\begin{tabular}{|l|l|l|l|}
\hline Measurement & Intra-observer I reliability & Intra-observer II reliability & $\begin{array}{l}\text { Inter-observer } \\
\text { reliability }\end{array}$ \\
\hline Post-op. PTS & 0.93 & 0.90 & 0.91 \\
\hline Pre-op. PTS & 0.88 & 0.90 & 0.82 \\
\hline TCCA $\left(^{\circ}\right)$ & 0.87 & 0.94 & 0.89 \\
\hline Tibia length & 0.99 & 0.99 & 0.99 \\
\hline
\end{tabular}

Table 1. The intraclass correlation coefficient analysis of the measured data. PTS posterior tibial slope, TCCA tibial component coronal alignment angle.

preoperative PTS, preoperative and postoperative KSS as well as postoperative ROM with postoperative PTS. $\mathrm{P}<0.05$ was considered to be statistically significant.

\section{Results}

The Kolmogorov-Smirnov test revealed that all data followed a normal distribution pattern. ICC and interclass correlation coefficients for the reproducibility of all parameters were $>80 \%$ (Table 1 ).

There were no statistically significant differences among the groups with regards to the demographic characteristics of patients before surgery, except that the age of the U2 Knee group was greater than the other groups (Table 2). There were also no statistically significant differences among the four groups with regards to preoperative and postoperative KSS and ROM (Table 2). The homogeneity of variance test indicated that there was no statistically significant difference among the corresponding data of each group (all P-values $>0.05$ ) (Table 3).

For the preoperative PTS and the tibia length, there were no significant differences among the four groups (all P-values $>0.05)$. For postoperative PTS, the Legion group had a significantly smaller slope than the U2 Knee and Vanguard groups $(\mathrm{P}=0.001)$ (Fig. 9). However, there was no significant difference in postoperative PTS between the Legion and NexGen groups $(\mathrm{P}=0.08)$, and no significant differences were found among the NexGen, U2 Knee, and Vanguard groups (all P-values $>0.05$ ).

There was no significant difference in TCCA among the four groups (all P-values $>0.05$ ). However, a one sample t-test was performed for the TCCAs of each group and $90^{\circ}$, and only the NexGen group showed no significant difference from $90^{\circ}(\mathrm{t}=-1.421, \mathrm{P}=0.162)$. Furthermore, the other prosthetic instruments and their PTS showed significant differences (all $\mathrm{P}<0.05)$ (Table 4).

Using the conventional tibial bone resection technology of two fingers proximally and three fingers distally. Empirically, it added about 3 degrees of posterior tilt. Therefore, A one sample t-test was performed for the 


\begin{tabular}{|l|l|l|l|l|l|}
\hline & Legion & U2 knee & NexGen & Vanguard & \\
\hline Age $($ years $)$ & $66.0 \pm 7.8$ & $70.9 \pm 6.1$ & $64.7 \pm 7.3$ & $63.7 \pm 7.7$ & $<0.001^{*}$ \\
\hline Gender $(\mathrm{m} / \mathrm{f})$ & $24 / 65$ & $19 / 49$ & $11 / 29$ & $4 / 12$ & 0.996 \\
\hline Side $(\mathrm{l} / \mathrm{f})$ & $45 / 59$ & $39 / 37$ & $24 / 22$ & $14 / 7$ & 0.230 \\
\hline Height $(\mathrm{cm})$ & $157.9 \pm 10.5$ & $157.2 \pm 9.4$ & $159.5 \pm 9.2$ & $158.3 \pm 9.0$ & 0.670 \\
\hline Weight $(\mathrm{kg})$ & $64.1 \pm 11.1$ & $62.9 \pm 9.7$ & $65.7 \pm 10.1$ & $63.6 \pm 9.4$ & 0.555 \\
\hline BMI $\left(\mathrm{kg} / \mathrm{m}^{2}\right)$ & $25.5 \pm 2.3$ & $25.3 \pm 2.5$ & $25.2 \pm 4.1$ & $25.2 \pm 2.1$ & 0.962 \\
\hline Pre-op. KSS & \multicolumn{5}{|l}{} \\
\hline Knee score & $37.4 \pm 11.4$ & $35.5 \pm 11.9$ & $38.1 \pm 11.1$ & $37.1 \pm 12.0$ & 0.619 \\
\hline Function score & $41.3 \pm 9.4$ & $39.1 \pm 10.3$ & $40.1 \pm 11.2$ & $37.3 \pm 12.4$ & 0.300 \\
\hline Post-op. KSS & \multicolumn{7}{|l|}{} \\
\hline Knee score & $90.2 \pm 4.1$ & $90.1 \pm 5.4$ & $89.9 \pm 5.3$ & $90.8 \pm 5.4$ & 0.899 \\
\hline Function score & $85.0 \pm 8.3$ & $85.1 \pm 8.4$ & $87.3 \pm 8.8$ & $85.2 \pm 9.8$ & 0.456 \\
\hline Pre-op. ROM & $74.2 \pm 18.2$ & $75.3 \pm 15.9$ & $73.4 \pm 16.4$ & $74.2 \pm 16.6$ & 0.938 \\
\hline Post-op. ROM & $112.4 \pm 12.5$ & $111.9 \pm 12.2$ & $113.1 \pm 15.5$ & $111.5 \pm 14.7$ & 0.959 \\
\hline
\end{tabular}

Table 2. Summary of patient and clinical results. BMI body mass index, KSS Knee Society score, ROM range of motion. ${ }^{\star} \mathrm{U} 2$ Knee was significantly different from those of other groups.

\begin{tabular}{|l|l|l|l|l|l|}
\hline Group & $\mathbf{n}$ & Pre-op. PTS $\left(^{\circ}\right)$ & Post-op. PTS $\left(^{\circ}\right)$ & TCCA $\left(^{\circ}\right)$ & Tibia length $(\mathbf{c m})$ \\
\hline Legion & 104 & $11.6 \pm 4.7$ & $6.3 \pm 2.6$ & $89.5 \pm 1.8$ & $34.2 \pm 2.5$ \\
\hline U2 knee & 76 & $10.8 \pm 4.9$ & $7.6 \pm 2.6$ & $89.5 \pm 1.9$ & $34.0 \pm 2.6$ \\
\hline NexGen & 46 & $10.0 \pm 5.8$ & $7.1 \pm 2.7$ & $89.6 \pm 1.8$ & $35.0 \pm 2.3$ \\
\hline Vanguard & 21 & $11.0 \pm 4.4$ & $8.6 \pm 3.3$ & $88.5 \pm 2.6$ & $34.8 \pm 2.6$ \\
\hline P & & 0.628 & 0.768 & 0.088 & 0.742 \\
\hline
\end{tabular}

Table 3. Measurement data and homogeneity of variance test of four kinds of prosthesis instrumentation. PTS posterior tibial slope, TCCA tibial component coronal alignment angle.

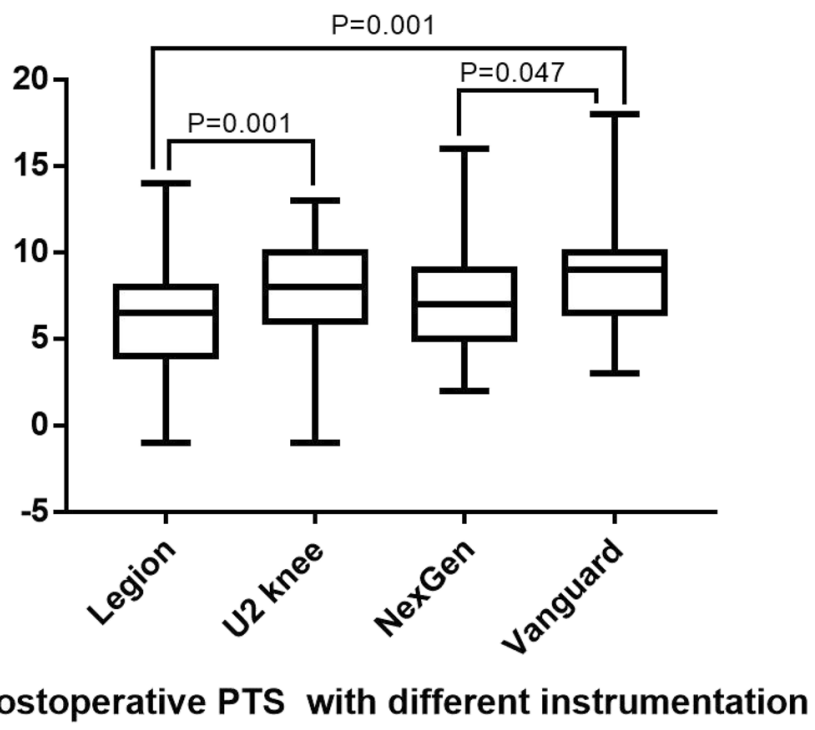

Figure 9. A boxplot illustrating the distributions of the four postoperative PTS.

postoperative PTS of each group and the fixed angle provided by the prosthetic instrumentations plus $3^{\circ}$, and the results indicated that only the Legion group $(t=1.199, \mathrm{P}=0.233)$ and the U2 Knee group $(\mathrm{t}=-0.998, \mathrm{P}=0.321)$ showed no statistical difference from the built-in posterior tilt angle provided by the product, whereas the PTS of the other prosthetic instrumentations were significantly different their specific fixed angles $($ all $\mathrm{P}<0.05)($ Table 4$)$.

Multiple linear regression showed that the tibial length and preoperative PTS had statistically significant effect on postoperative PTS $(b=-0.023$ and $0.093, t=-3.474$ and $2.679, P=0.001$ and 0.008 , respectively) (Table 5); 


\begin{tabular}{|l|l|l|r|r|l|l|l|l|}
\hline Group & Post-op. PTS $\left(^{\circ}\right)$ & Fixed angle + 3 $\left(^{\circ}\right)$ & t & P & TCCA $\left(^{\circ}\right)$ & $\mathbf{9 0 ^ { \circ }}$ & t & P \\
\hline Legion & $6.3 \pm 2.6$ & 6 & 1.199 & $0.233^{*}$ & $89.5 \pm 1.8$ & 90 & -2.337 & 0.021 \\
\hline U2 Knee & $7.6 \pm 2.6$ & 8 & -0.998 & $0.321^{*}$ & $89.5 \pm 1.9$ & 90 & -2.213 & 0.030 \\
\hline NexGen & $7.1 \pm 2.7$ & 10 & -6.967 & $<0.001$ & $89.6 \pm 1.8$ & 90 & -1.421 & $0.162^{*}$ \\
\hline Vanguard & $8.6 \pm 3.3$ & 3 & 7.644 & $<0.001$ & $88.5 \pm 2.6$ & 90 & -2.464 & 0.023 \\
\hline
\end{tabular}

Table 4. One-sample t-test results for the postoperative PTS compared with the fixed angle provided by the cutting block plus $3^{\circ}$ and between the TCCAs and $90^{\circ}$. PTS posterior tibial slope, TCCA tibial component coronal alignment angle. ${ }^{\star} \mathrm{P}>0.05$.

\begin{tabular}{|c|c|c|c|c|c|}
\hline Independent variable & Unstandardized coefficient b & Standard error & Standardized coefficient $b$ & t value & P Value \\
\hline Constant & 19.466 & 9.095 & - & 2.140 & 0.033 \\
\hline Pre-op. PTS & 0.093 & 0.035 & 0.165 & 2.679 & $0.008^{*}$ \\
\hline TCCA & -0.129 & 0.087 & -0.089 & -1.484 & 0.139 \\
\hline Tibia length & -0.023 & 0.007 & -0.212 & -3.474 & $0.001^{\star}$ \\
\hline BMI & -0.076 & 0.062 & -0.074 & -1.230 & 0.220 \\
\hline Post-op. KSS (knee score) & 0.046 & 0.070 & 0.080 & 0.664 & 0.507 \\
\hline Post-op. KSS (function score) & -0.008 & 0.037 & -0.023 & -0.204 & 0.839 \\
\hline Post-op. ROM & 0.041 & 0.022 & 0.191 & 1.848 & 0.066 \\
\hline
\end{tabular}

Table 5. Multiple linear regression of influencing factors on postoperative PTS. Dependent variable: post-op. PTS. PTS posterior tibial slope, TCCA tibial component coronal alignment angle, BMI body mass index, KSS Knee Society score, $R O M$ range of motion. ${ }^{*} \mathrm{p}<0.05$.

although, there were weak correlations between the tibial length and postoperative PTS $(\mathrm{R}=0.255, \mathrm{P}<0.001)$, and between preoperative PTS and postoperative PTS $(\mathrm{R}=0.210, \mathrm{P}=0.001)$. The different ages, TCCAs, BMI, postoperative KSS, and ROM had no significant effect on PTS (Table 5).

\section{Discussion}

The PTS is defined as the angle between the tangent of the medial and lateral plateaus and the line perpendicular to the longitudinal mechanical axis ${ }^{14}$. This angle is very different between individuals and ranges in studies from $-9^{\circ}$ to $16^{\circ}$ with an average of approx. $3^{\circ}-10^{\circ 16-18}$.

Currently, the most commonly used method for measuring the PTS is the true lateral radiograph of the tibia. For optimal determination, a strictly lateral radiograph of the entire length of the tibia, including the ankle and knee joint, is required ${ }^{14}$. The mechanical axis (TLA) is constructed from a line between the center of the tibial plateau and that of the lateral ankle joint. A tangent is placed over the tibial plateau, and the angle between the mechanical axis and the tangent determines the PTS. However, in clinical practice, before and after TKA, fulllength lateral radiographs of the tibia are not routinely available, so there are many alternatives for the tibial mechanical axis, such as the tibial proximal anatomical axis ${ }^{18-22}$, tibial shaft anatomical axis ${ }^{18,23}$, posterior tibial $\operatorname{cortex}^{18,20,22}$, anterior tibial cortex ${ }^{18-20,22}$, and fibular shaft axis ${ }^{18,23}$. Compared with the tibial mechanical axis, the accuracy of the PTS measurements varied. Current studies show there is good correlation between the tibial proximal anatomical axis, constructed by measuring points $5-15 \mathrm{~cm}$ or $6-16 \mathrm{~cm}$ below the joint surface, and the mechanical axis. In this way, deviations can be reduced to up to $1.5^{\circ 14,18}$. The shorter the radiograph that includes the tibia, the worse the PTS measurement accuracy tends to be in these studies. Therefore, based on the results of these studies, we used an expanded lateral radiograph of a at least $20-\mathrm{cm}$ long section of the tibia to measure PTS in this study. Obviously, the accuracy of this measurement is lower than that obtained when using the full-length lateral tibia, which is a limitation of this study. However, according to the literature ${ }^{14,18}$, this was the alternative method with the smallest error.

Appropriate PTS for TKA is very important. Previous studies suggested that postoperative PTS should range from $0^{\circ}$ to $10^{\circ}$ to guarantee optimal prosthetic function. Excessive PTS after TKA may cause anterior and posterior instability, leading to anterior subluxation of the tibia, thus increasing the shear stress of the posterior tibia polyethylene and resulting in aseptic loosening ${ }^{22}$. Conversely, a reduction in the PTS leads to increased stress in the anterior part of the subchondral bone, thereby increasing the risk of component subsidence ${ }^{24}$. Decreased PTS also leads to limited flexion because of the tight flexion gap ${ }^{25}$.

The method used for tibial bone resection primarily depends on the implant instrumentation provided by the manufacturer. In the coronal plane, the tibial bone resection needs to be perpendicular to the tibial mechanical axis. In addition to navigation and patient-specific instrumentation, the traditional method of aligning the tibial mechanical axis is to use the proximal spike of the cutting guide to anchor the ACL attachment to the tibia ${ }^{26}$, the anterior middle third of the anterior and posterior axis of the tibial plateau ${ }^{27}$, and the intercondylar eminentia ${ }^{28}$, resulting in the extramedullary rod being parallel to the palpable fibula ${ }^{27}$. In the distal tibia, because the ankle joint center is difficult to locate, the second metatarsal ${ }^{29}$, first and second metatarsal spaces ${ }^{30}$, tibialis anterior 
tendon, or anterior tibial crest ${ }^{31,32}$ are often used as markers, and through these, generally good coronal alignment can be obtained. The sagittal mechanical axis of the tibia is more difficult to mark than the coronal mechanical axis. Therefore, the specific method of bone resection of the PTS is still controversial, and there is no unified standard. The traditional method is to adjust the PTS using the distance between the tibial cutting guide rod and the anterior skin surface of the tibia as a reference ${ }^{33}$. The accuracy of the cutting block with a fixed angle posterior slope provided by the manufacturer was uncertain. Therefore, the present study used conventional tibial bone resection techniques to compare the actual PTS obtained by various makes of cutting blocks with fixed angles to test their accuracy. We found that when we used a $3^{\circ}$ cutting block (Legion), the angle after bone resection was $6.3^{\circ} \pm 2.6^{\circ}$. For a $5^{\circ}$ cutting block (U2 Knee), the angle after osteotomy was $7.6^{\circ} \pm 2.6^{\circ}$. With the $7^{\circ}$ cutting block, the angle after osteotomy was $7.1^{\circ} \pm 2.7^{\circ}$ (NexGen). Surprisingly, for the Vanguard's $0^{\circ}$ cutting block, the angle after resection was $8.6^{\circ} \pm 3.3^{\circ}$. We performed multiple linear regression analysis of the PTS with the parameters of age, TCCA, BMI, preoperative PTS, postoperative KSS, ROM, and tibial length, and found that only the tibial length and preoperative PTS affected the PTS, although, the effect was very small $(\mathrm{R}=0.255$ and 0.210 , respectively). However, considering that we used the expanded lateral radiograph with a $20-\mathrm{cm}$ section of the tibia instead of the full-length lateral tibial radiograph, the actual PTS may be $1^{\circ}-1.5^{\circ}$ less than the above value. In addition, in the coronal plane, we used the second metatarsal bone to align the cutting guide rod, and the TCCAs obtained with these four prosthetic instrumentations were $89.5^{\circ}-89.6^{\circ}$; only the NexGen group had no statistical difference from $90^{\circ}$, while the other three groups all showed statistical difference from $90^{\circ}$. This suggests that the second metatarsal bone is not a reliable reference marker for coronal tibial bone resection with some instrumentations, as it is more easily affected by the position of the ankle joint.

There were several limitations to our study. First, different surgeons have different finger widths, and using this traditional surgical measurement technique may cause the distance between tibial cutting guide rod and the anterior edge of the tibia to be different between different surgeons, resulting in different PTS. Although all operations in this study were performed by the same surgeon, which minimized the bias caused by the surgical technique, the study did not consider the influence of the finger widths of different surgeons on the tibial osteotomy. Second, because of the use of the extended tibial lateral radiograph instead of full-length lateral radiograph of the tibia, the PTS obtained was reduced by an average of 1.5 degrees. Even so, the posterior tibia obtained by using Legion, U2 Knee, and Vanguard instrumentations were all greater than the angles of the fixed angles on the cutting block, which means that the PTS obtained using these four instrumentations were greater than those using the cutting block. However, the angle after resection for the Vanguard's $0^{\circ}$ cutting block was $8.6^{\circ} \pm 3.3^{\circ}$ and thus had a significantly greater margin of error than the other three instrumentations. The reasons may be that (1) the sample size was too small, thus a larger sample is needed for future verification, and (2) the anatomical features of the proximal tibia may differ between East Asians and Caucasians, and knee deformity is usually more pronounced in Asian than Caucasian patients at the time of TKA. Nevertheless, according to the postoperative KSS and knee ROM, most of the patients obtained satisfactory results. Therefore, although the PTS is not completely consistent with the angle of the cutting block, using conventional tibial bone resection technology with different tibial cutting instrumentations provided by various manufacturers in TKA can obtain safe PTS.

Received: 23 August 2020; Accepted: 16 March 2021

Published online: 31 March 2021

\section{References}

1. Johnston, H., Abdelgaied, A., Pandit, H., Fisher, J. \& Jennings, L. M. The effect of surgical alignment and soft tissue conditions on the kinematics and wear of a fixed bearing total knee replacement. J. Mech. Behav. Biomed. Mater. 100, 103386. https://doi.org/10. 1016/j.jmbbm.2019.103386 (2019).

2. Klatt, B. A., Goyal, N., Austin, M. S. \& Hozack, W. J. Custom-fit total knee arthroplasty (OtisKnee) results in malalignment. J. Arthroplasty. 23, 26-29. https://doi.org/10.1016/j.arth.2007.10.001 (2008).

3. Fujito, T. et al. Influence of posterior tibial slope on kinematics after cruciate-retaining total knee arthroplasty. J. Arthroplasty. 33, 3778-3782. https://doi.org/10.1016/j.arth.2018.07.029 (2018).

4. Kang, K. T. et al. Biomechanical effects of posterior condylar offset and posterior tibial slope on quadriceps force and joint contact forces in posterior-stabilized total knee arthroplasty. Biomed. Res. Int. 2017, 4908639. https://doi.org/10.1155/2017/4908639 (2017).

5. O’Rourke, M. R., Callaghan, J. J., Goetz, D. D., Sullivan, P. M. \& Johnston, R. C. Osteolysis associated with a cemented modular posterior-cruciate-substituting total knee design : Five to eight-year follow-up. J. Bone Joint Surg. Am. 84, 1362-1371. https://doi. org/10.2106/00004623-200208000-00011 (2002).

6. Karas, V. et al. Total knee arthroplasty in patients less than 50 years of age: results at a mean of 13 years. J. Arthroplasty. 34, 2392-2397. https://doi.org/10.1016/j.arth.2019.05.018 (2019).

7. Okamoto, S. et al. Effect of tibial posterior slope on knee kinematics, quadriceps force, and patellofemoral contact force after posterior-stabilized total knee arthroplasty. J. Arthroplasty. 30, 1439-1443. https://doi.org/10.1016/j.arth.2015.02.042 (2015).

8. Bai, B., Baez, J., Testa, N. \& Kummer, F. J. Effect of posterior cut angle on tibial component loading. J. Arthroplasty. 15, 916-920. https://doi.org/10.1054/arth.2000.9058 (2000).

9. Dorr, L. D. \& Boiardo, R. A. Technical considerations in total knee arthroplasty. Clin. Orthop. Relat. Res. 205, 5-11 (1986).

10. Ewald, F. C. et al. Kinematic total knee replacement. J. Bone Joint Surg. Am. 66, 1032-1040 (1984).

11. Chambers, A. W., Wood, A. R., Kosmopoulos, V., Sanchez, H. B. \& Wagner, R. A. Effect of posterior tibial slope on flexion and anterior-posterior tibial translation in posterior cruciate-retaining total knee arthroplasty. J. Arthroplasty. 31, 103-106. https:// doi.org/10.1016/j.arth.2015.08.027 (2016).

12. Kang, K. T. et al. The increase in posterior tibial slope provides a positive biomechanical effect in posterior-stabilized total knee arthroplasty. Knee Surg. Sports Traumatol. Arthrosc. 26, 3188-3195. https://doi.org/10.1007/s00167-018-4925-3 (2018).

13. Singh, G. et al. Restoring the anatomical tibial slope and limb axis may maximise post-operative flexion in posterior-stabilised total knee replacements. Bone Joint J. 95-B, 1354-1358. https://doi.org/10.1302/0301-620X.95B10.31477 (2013).

14. Faschingbauer, M., Sgroi, M., Juchems, M., Reichel, H. \& Kappe, T. Can the tibial slope be measured on lateral knee radiographs?. Knee Surg. Sports Traumatol. Arthrosc. 22, 3163-3167. https://doi.org/10.1007/s00167-014-2864-1 (2014).

15. Landis, J. R. \& Koch, G. G. The measurement of observer agreement for categorical data. Biometrics 33, 159-174 (1977). 
16. Lombardi, A. V. Jr., Berend, K. R., Aziz-Jacobo, J. \& Davis, M. B. Balancing the flexion gap: Relationship between tibial slope and posterior cruciate ligament release and correlation with range of motion. J. Bone Joint Surg. Am. 90(Suppl 4), 121-132. https://doi. org/10.2106/JBJS.H.00685 (2008).

17. Nunley, R. M., Nam, D., Johnson, S. R. \& Barnes, C. L. Extreme variability in posterior slope of the proximal tibia: Measurements on 2395 CT scans of patients undergoing UKA?. J. Arthroplasty. 29, 1677-1680. https://doi.org/10.1016/j.arth.2014.03.024 (2014).

18. Yoo, J. H., Chang, C. B., Shin, K. S., Seong, S. C. \& Kim, T. K. Anatomical references to assess the posterior tibial slope in total knee arthroplasty: A comparison of 5 anatomical axes. J. Arthroplasty. 23, 586-592. https://doi.org/10.1016/j.arth.2007.05.006 (2008).

19. Bae, D. K., Song, S. J., Yoon, K. H., Noh, J. H. \& Moon, S. C. Comparative study of tibial posterior slope angle following cruciateretaining total knee arthroplasty using one of three implants. Int. Orthop. 36, 755-760. https://doi.org/10.1007/s00264-011-1395-3 (2012).

20. Pan, X. Q. et al. Effect of tibial slope changes on femorotibial contact kinematics after cruciate-retaining total knee arthroplasty. Knee Surg Sports Traumatol. Arthrosc. 25, 3549-3555. https://doi.org/10.1007/s00167-016-4384-7 (2017).

21. Utzschneider, S. et al. Development and validation of a new method for the radiologic measurement of the tibial slope. Knee Surg Sports Traumatol. Arthrosc. 19, 1643-1648. https://doi.org/10.1007/s00167-011-1414-3 (2011).

22. Zhang, Y. et al. Measurement and comparison of tibial posterior slope angle in different methods based on three-dimensional reconstruction. Knee 21, 694-698. https://doi.org/10.1016/j.knee.2014.01.008 (2014).

23. Han, H. S., Chang, C. B., Seong, S. C., Lee, S. \& Lee, M. C. Evaluation of anatomic references for tibial sagittal alignment in total knee arthroplasty. Knee Surg. Sports Traumatol. Arthrosc. 16, 373-377. https://doi.org/10.1007/s00167-008-0486-1 (2008).

24. Hofmann, A. A., Bachus, K. N. \& Wyatt, R. W. Effect of the tibial cut on subsidence following total knee arthroplasty. Clin. Orthop. Relat. Res. 269, 63-69 (1991).

25. Jojima, H., Whiteside, L. A. \& Ogata, K. Effect of tibial slope or posterior cruciate ligament release on knee kinematics. Clin. Orthop. Relat. Res. 426, 194-198. https://doi.org/10.1097/01.blo.0000138960.57680.60 (2004).

26. Reed, M. R. et al. Extramedullary or intramedullary tibial alignment guides: A randomised, prospective trial of radiological alignment. J. Bone Joint Surg. Br. 84, 858-860. https://doi.org/10.1302/0301-620x.84b6.12702 (2002).

27. Laskin, R. S. Instrumentation pitfalls: you just can't go on autopilot!. J. Arthroplasty. 18, 18-22. https://doi.org/10.1054/arth.2003. 50066 (2003).

28. Bek, D., Ege, T., Yıldız, C., Tunay, S. \& Başbozkurt, M. The accuracy of two different extra-medullary tibial cutting guides for posterior tibial slope in total knee arthroplasty. Eklem Hastalik Cerrahisi. 25, 75-79. https://doi.org/10.5606/ehc.2014.17 (2014).

29. Tsukeoka, T., Tsuneizumi, Y. \& Lee, T. H. Accuracy of the second metatarsal as a landmark for the extramedullary tibial cutting guide in total knee arthroplasty. Knee Surg. Sports Traumatol. Arthrosc. 22, 2969-2974. https://doi.org/10.1007/s00167-014-3254-4 (2014).

30. Tew, M. \& Waugh, W. Tibiofemoral alignment and the results of knee replacement. J. Bone Joint Surg. Br. 67, 551-556 (1985).

31. Sasanuma, H., Sekiya, H., Takatoku, K., Ajiki, T. \& Hagiwara, H. Accuracy of a proximal tibial cutting method using the anterior tibial border in TKA. Eur. J. Orthop. Surg. Traumatol. 24, 1525-1530. https://doi.org/10.1007/s00590-014-1415-2 (2014).

32. Tsukeoka, T., Lee, T. H., Tsuneizumi, Y. \& Suzuki, M. The tibial crest as a practical useful landmark in total knee arthroplasty. Knee. 21, 283-289. https://doi.org/10.1016/j.knee.2012.09.002 (2014).

33. Tsukeoka, T. \& Tsuneizumi, Y. The distance from the extramedullary cutting guide rod to the skin surface as a reference guide for the tibial slope in total knee arthroplasty. Knee. 23, 314-317. https://doi.org/10.1016/j.knee.2015.09.005 (2016) ((epub 2016 Jan 12)).

\title{
Acknowledgements
}

The researchers acknowledge the support of the Imaging Center of Honghui Hospital, Xian Jiaotong University.

\section{Author contributions}

Y.L. performed the measurement, analyzed the data and wrote the manuscript. X.Y. performed the measurement collected the data. F.Q. processed the data. Y.H. supervised the whole study process, and helped to review the manuscript. All authors have read and approved the manuscript.

\section{Funding}

This study was supported by a grant from Shaanxi Provincial Key Research and Development Project (CN) (Grant no. 2019SF-214) and Xi'an Health Scientific Research Talent Project(CN) (Grant no. J201903058). The authors have no conflicts of interest to declare.

\section{Competing interests}

The authors declare no competing interests.

\section{Additional information}

Correspondence and requests for materials should be addressed to Y.H.

Reprints and permissions information is available at www.nature.com/reprints.

Publisher's note Springer Nature remains neutral with regard to jurisdictional claims in published maps and institutional affiliations.

\begin{abstract}
Open Access This article is licensed under a Creative Commons Attribution 4.0 International License, which permits use, sharing, adaptation, distribution and reproduction in any medium or format, as long as you give appropriate credit to the original author(s) and the source, provide a link to the Creative Commons licence, and indicate if changes were made. The images or other third party material in this article are included in the article's Creative Commons licence, unless indicated otherwise in a credit line to the material. If material is not included in the article's Creative Commons licence and your intended use is not permitted by statutory regulation or exceeds the permitted use, you will need to obtain permission directly from the copyright holder. To view a copy of this licence, visit http://creativecommons.org/licenses/by/4.0/.
\end{abstract}

(C) The Author(s) 2021 